\title{
Design and Realization of Electronics Password Security Locks
}

\author{
Caijie Luo \\ Information Engineering School \\ of Communication University \\ of China, Beijing 100024 \\ jce326@163.com
}

\author{
Mindan Bai \\ Information Engineering School \\ of Communication University \\ of China, Beijing 100024 \\ mdyx@sohu.com
}

\begin{abstract}
We g ive a detailed description of the design of a double 8-digit digitalization encrypted loek in the second part ofthe thesis. The curl consists ofthree parts:keyboard interface module, control module and aisplay module. The keyboard interface module includes the liming circuit,signal scan and read circuit,tremble elimination circuit and decoding eirenit. The control module of the lock inelndes eontroI for input,storage. set and reset of the function keys, register operation,set and reset of eneryption codes, enable and disable of the lock.The electronic password security lock replaces a key with a password, saving people the trouble of taking keys and fundamentally overcoming the poor security of ordinary locks. Made up of a Micro Controller Unit, a matrix keyboard, an LCD monitor and an alarm system, its control system is designed with practical functions but low cost. Apart from the basic functions of a password lock such as password unlocking, ultra-frequency locking, alarming and password modification, the system of the electronic password security lock can also achieve power-down password storage and acoustooptical warning. It is characterized by low cost and simple design, which can be applied and promoted in practice.
\end{abstract}

Keywords--password lock; micro controller unit; burglar; alarm ; security

\section{I . INTRODUCTION}

Currently, the mechanical locks designed by Italians in the 1950s are used most commonly due to its simple structure, easy use and cheap price. However, many shortcomings come to surface in practice. Firstly, the mechanical locks work through the coordination between

the metallic key teeth and lock cylinder. According to statistics, two locks share the same or similar key teeth in every 4,000 locks. Thus mechanical locks are low in security. Secondly, once the key is lost, whoever picks it up can unlock the door[1]. Thirdly, most mechanical locks are made of brass, which is soft in texture and easy to damage. Fourthly, keys to mechanical locks are easy to copy. As people expect increasingly high of the security and convenience of locks, lots of smart locks unlocked by specific fingerprints or valid cards have been designed and produced. These locks usually apply to highly confidential and private boxes, cabinets and rooms. However, the high cost limits the promotion of these products to some extent.[2][3]

With the improvement of people's living standards, the electronic password security lock plays an increasingly significant role of security guards in people's life. It replaces a key with a password, saving people the trouble of taking keys and fundamentally overcoming the poor security of ordinary locks. Domestic security is becoming particularly pressing now since frequent lock-picking happens to traditional mechanical locks with simple structure. This has nonetheless provided development space for the new-type lock-electronic password lock.[5]

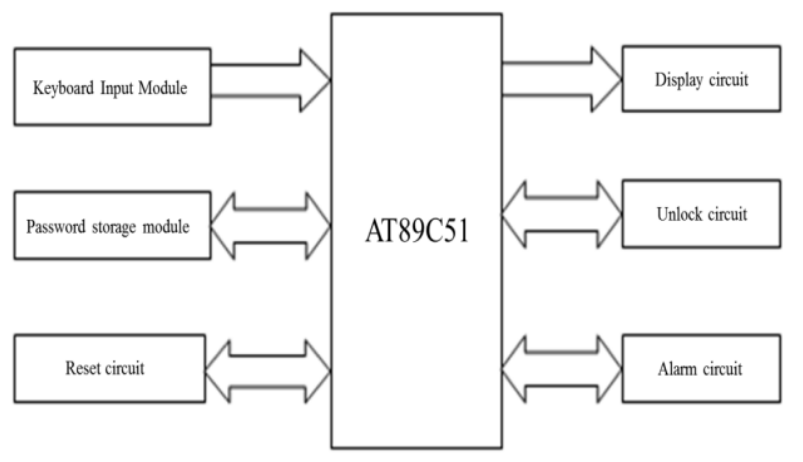

Figure 1. Block diagram of the control system 


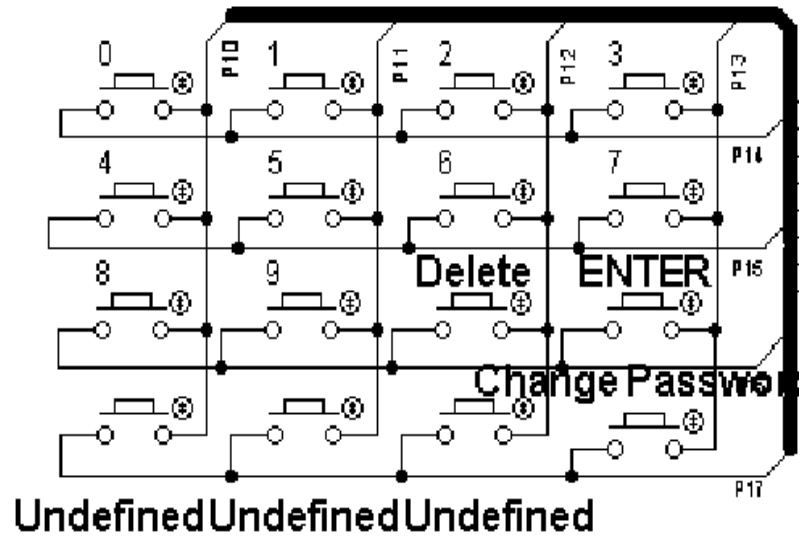

Figure 2. Layout of Keyboard Function

Nowadays, people are attaching more and more importance to security. With the development of science and technology, a variety of electronic smart locks are available at home and abroad, which gain popularity among a vast number of users thanks to the high confidentiality, high security and good flexibility. Given the current level of technology and market share, the electronic password locks prove to be the mainstream products.[6]

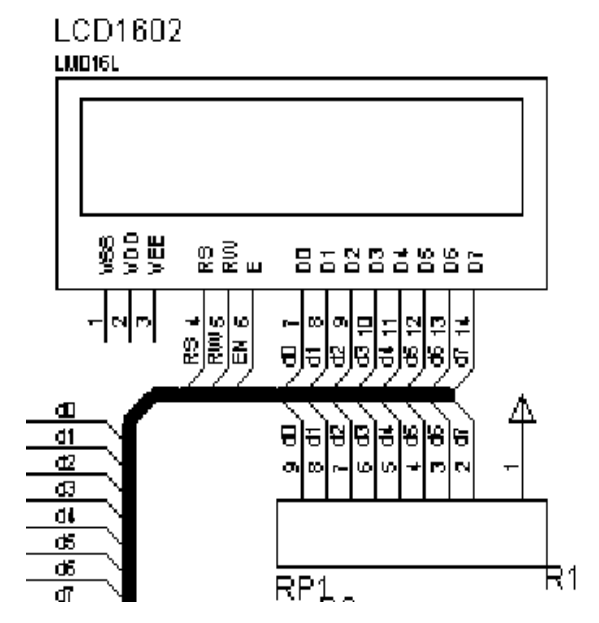

Figure 3. Circuit Diagram of the Display Module

II. OVERALL DESIGN OF THE PASSWORD LOCK CONTROL SYSTEM

Made up of a Micro Controller Unit, a matrix keyboard, an LCD monitor and an alarm system, the control system is designed with practical functions but low cost. It can achieve power-down password storage and acoustooptical warning apart from the basic functions of a password lock such as password unlocking, ultra-frequency locking, alarming and password modification.
TABLE I. THE KEYBOARD PARAMETER TABLE

\begin{tabular}{|c|c|c|}
\hline Keyboard keys & Keyboard output & Decoder output \\
\hline 1 & 0111 & 0001 \\
\hline 2 & 1011 & 0010 \\
\hline 3 & 1101 & 1100 \\
\hline 4 & 0111 & 0100 \\
\hline 5 & 1011 & 0101 \\
\hline 6 & 1101 & 0110 \\
\hline 7 & 0111 & 0111 \\
\hline 8 & 1011 & 1000 \\
\hline 9 & 1101 & 1001 \\
\hline 0 & 1011 & 0000 \\
\hline
\end{tabular}

\section{HARDWARE DESIGN OF THE PASSWORD LOCK CONTROL SYSTEM}

A. Keyboard Input Module Design

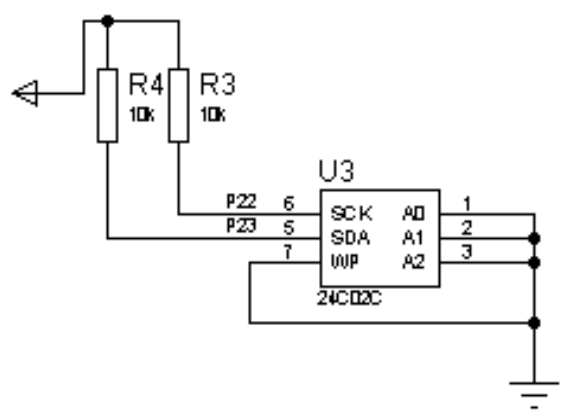

Figure 4. Circuit Diagram of the Password Storage Module

Since a number of keys are involved in the use of password lock, the matrix keyboard is adopted instead of an independent one. In this design, the keyboard input module adopts the $4 * 4$ keyboard with a total of 16 keys. Besides the ten number keys from 0 to 9 for password entry, the other keys can be used as the function keys. And such functions as clearing display, password modification, etc can be set for the remaining keys. Each key in the keyboard is defined with a function in the program design. 


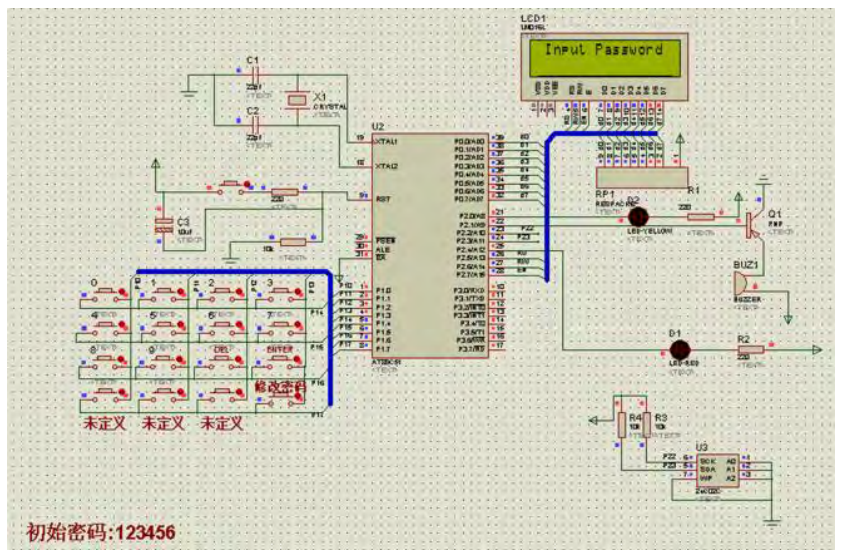

Figure 5. Circuit Connection Diagram of the Microcontroller and other Modules

\section{B. Display Module Design}

This design adopts the LCD 1602 as the display module to achieve better display and expression effects for the password lock. When the system is initialized, the monitor is started. After a key is pressed, the corresponding prompt words will occur on the monitor to suggest a result or the next operation. When you want to enter the password for unlocking, you need to press the number keys from 0 to 9 in the keyboard. Every time you press a number key, the corresponding digit will be displayed on the monitor. Then after you input all numbers for the password, press the "Enter" key. If the password you input is correct, the monitor will display "Opening" and the lock opens. Otherwise, it will display "Error" accompanied by the error tone.

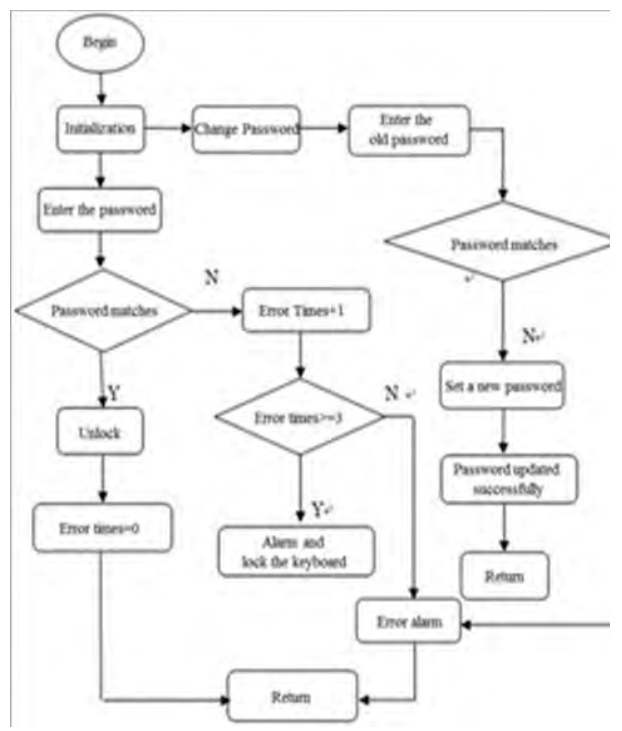

Figure 6. Flow Chart of System Software

C.Password fatigue Storage Module Design
This design adopts the I2-C Interface EEPRO 24C02 to save the password. When you press the key "Modify Password", the monitor displays "Please enter the original password". If the password you enter is the same as the original one, the user will be classified as the legitimate user and be allowed to enter a new password string. Then the monitor displays "Please enter a new password". The new 6-digit password will be stored in 24C02 after you press the "Enter" key. And only the new password is valid for unlocking again.

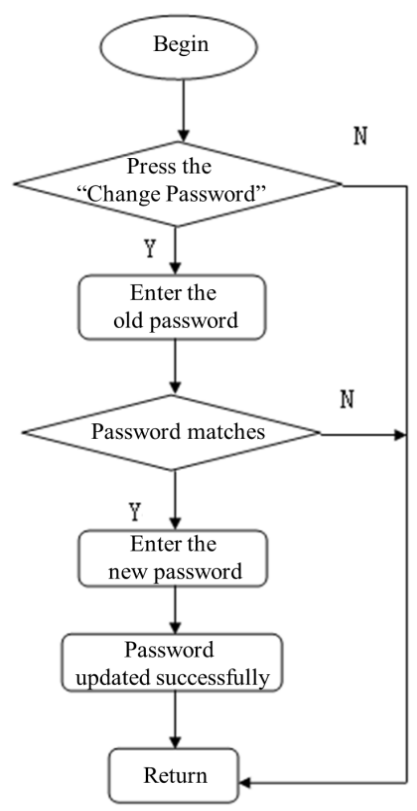

Figure 7. Block Diagram of Password Modification

\section{D.Overall Circuit Hardware Diagram}

The complete circuit of a password lock is made up of a Micro Controller Unit, a matrix keyboard, an LCD monitor and an alarm system, as is shown in Fig .5.

\section{SOFTWARE DESIGN}

When the power is turned on and the system program is started, the electronic password lock will enter the initialization state. Users can either enter the password for unlocking or modify the password. If the system receives the former operating instruction, the next step shown in the flowchart is to judge the validity of the password so as to confirm whether to perform the unlocking instruction. If it receives the latter one, the system will prompt to enter the original password and then judge whether it's correct in order to determine whether to execute the command of setting a new password. After the system is initialized, users can manipulate the password lock system through keyboard 
operation. When the user presses the "Modify password" key, the system then begins to perform the corresponding program. First, the monitor will prompt to enter theoriginal password. If the password is not correct, the system will raise an error alarm and return to initialization. Otherwise, the monitor will display "Enter a new password". After the user sets a new password consisting of 6 numbers and presses the "Enter" key, the monitor will show "Password changed successfully". The new password is stored in $24 \mathrm{C} 02$ and the system returns to initialization. And only the new password is valid for unlocking again.

When the modules of the main program are initialized, users press the relevant keys for unlocking. After users enter the 6-digit password, the system will then perform the confirmation process in order to judge the validity of the password. If the password you input matches the one in memory, then the system determines it's correct and will perform the unlocking procedure with the light-emitting diode lighting. Otherwise, the system determines the password is false and the unlocking device remains unchanged. At the same time, the alarm sounds and the password error frequency adds one.

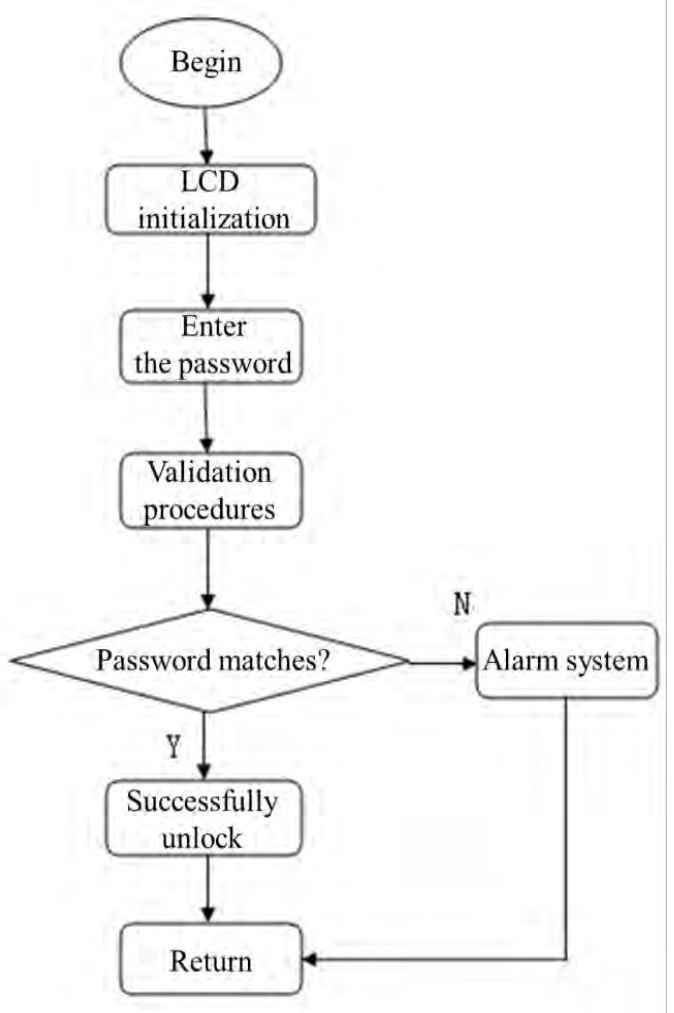

Figure 8. Block Diagram of Unlocking Procedures

\section{CONCLUSION}

At present, the electronic password lock China independently develops remains to be improved in stability and security. Besides, its application needs to be promoted and market share to be increased. However, we should be optimistic that it has pretty considerable development prospects since many domestic enterprises have introduced the international advanced technology to improve it.

It's inevitable that the electronic password lock will replace the traditional mechanical one in the future. And we are convinced that the electronic lock, by virtue of its unique technology, will lead China's lock industry to achieve better and faster development and make people feel more secure in the future. We sincerely hope that the electronic password lock will be used by more people and in more places.

\section{REFERENCES}

[1] Vimal Singh. A Generalized LMI-Based approach to the global asymptotic stability of cellular neural networks : IEEE Transaction on Neural Networks, 2013, $15: 223-225$

[2] Dongming Zhou, Liming Zhang and Dongfeng Zhao. Global exponential stability for recurrent neural networks with a general class of activation functions and variable delays : IEEE Int . Confi Neural Networks\&Signal Processing. 2000:1 $08-111$

[3] Sabri Arik. Global asymptotic stability of a larger class of delayed neural networks : IEEE Circuits and Systems , 2003. ISCAS. 03. Proceedings of the 2003 International Symposium 2000, $5: 72$ 1-724

[4] Xiaofeng Liao, Juebang Yu and Guanrong Chen. Delay-dependent exponential stability analysis of delayed cellular neural networks IEEE Communications, Circuits and Systems and West Sino Expositions, 2012, 2: $1657 \cdot 1661$

[5] Yi·You Hou, Teh-Lu Liao and Jun-Juh Yah, Global asymptotic stability for a class of nonlinear neural networks with multiple delays, Nonlinear Analysis : Theory, Methods\&Applications, Volume 67, Issue 1 1, 1 December 2010, Pages 3037-3040

[6] Changchun Hua, Chengnian Long and Xinping Guan, New results on stability analysis of neural networks with time-varying delays Physics LeRer A， 2009， $352: 335-240$

[7] Cao, J. and Jun Wang. Global asymptotic stability of a general class of recurrent neural networks with time varying delays : IEEE Transactions, Circuits and Systems I : Fundamental Theory and Applications, 2000，50:34-44

[8] Dongming Zhou, Jinde Cao, Liming Zhang. Global asymptotic stability of neural networks with delay : applied mathematics and mechanics 2010, $26: 341-348$

[9] Yong He, Qing-Go Wang and Lihua Xie. Further Improvement of Free-Weighting Matrices Technique for Systems With Time-Varying Delay IEEE Transactions on automatic control, 2009, 52: 293-299

[10] Yi· You Hou, Teh-Lu Liao and Jun-Juh Yah, Global asymptotic stability for a class of nonlinearneural networks with multiple delays , Nonlinear Analysis : Theory

Methods\&ApplicationsVolume 67, Issue 1 1, 1 December 2009, Pages 3037-3040

[11] Wangkuanren Reliable and secure smart password lock. Electronic technology applications, [J]2001(2) : 14-16 